\title{
MECANISMOS DA GOVERNANÇA DA APRENDIZAGEM ORGANIZACIONAL (GovA)
}

Giselly Rizzatti ${ }^{1}$ Patricia de Sá Freire ${ }^{2}$

\section{RESUMO}

Com o objetivo de diminuir a lacuna de estudos sobre Governança daAprendizagem (GovA), este artigo pretende descrever os mecanismos constitutivos da Governança da Aprendizagem sob a perspectiva organizacional. A pesquisa é de abordagem qualitativa, de cunho teórico, com objetivos exploratórios e descritivos, por meio bibliográfico, com apoio de uma revisão sistemática da literatura, respeitando-se os métodos da revisão integrativa. Como resultado foi possível descrever a análise do conhecimento científico produzido sobre GovA ao longo do tempo, de modo a traçar a sua evolução. Foi identificada a proposição de três mecanismos de GovA: a Governança do Conhecimento (GovC), a Gestão do Conhecimento (GC) e o Ciclo de Conhecimento. Com base na revisão sistemática realizada, pode-se afirmar que, nas bases de dados estudadas, esta pesquisa vem contribuir para a mitigação da lacuna de conhecimento sobre o tema.

Palavras-chave: Gestão do conhecimento. Aprendizagem organizacional. Governança do conhecimento. Governança da aprendizagem. 
A gestão do conhecimento e a aprendizagem organizacional tornaram-se essenciais para a sobrevivência das organizações por agregarvalor a produtos e marcas. Com isso, criou-se a necessidade de governar os esforços do ciclo de criação do conhecimento e, consequentemente, do ciclo da aprendizagem nas organizações (PEMSEL; MULLER, 2012). Porém, para governar os mecanismos e procedimentos que envolvem uma efetiva gestão do conhecimento e alcançar os objetivos estratégicos da organização, há a necessidade de dar suporte aos processos de conhecimento e aprendizagem, compreendendo os componentes, suas inter-relações e seu funcionamento propriamente dito.

Surge, então, uma nova abordagem de governança,intitulada Governança da Aprendizagem Organizacional (GovA), que ,segundo os autores Vitry e Chia (2015, p. 281) consiste nos: processos cognitivos e comportamentais que permitem aos atores e stakeholders produzirem uma visão compartilhada sobre a organização, criarem e desenvolverem uma organização coletiva e negociarem estratégias e objetivos comuns.

Naliteratura científica, os estudos sobre o termo emergem como forma de compreender o papel do conhecimento e da aprendizagem para o crescimento e desenvolvimento da organização, reunindo novas percepções teóricas e ênfases empíricas. Entretanto, mesmo sabendo-se da emergência de estudos sobre o construto, Belle (2016) explica que falta quantidade e maior aprofundamento dos estudos sobre a relação do termo governança e aprendizagem organizacional para alcançar os objetivos estratégicos e melhorar o desempenho da organização.

Neste contexto, com o objetivo maior de diminuir a lacuna de estudos sobre GovA, este artigo pretende descrever os mecanismos constitutivos da Governança da Aprendizagem sob a perspectiva organizacional.

\section{MECANISMOS DE GOVERNANÇA DA APRENDIZAGEM (GovA)}

Na última década, a importância estratégica do conhecimento tem sido cada vez mais reconhecida pelas organizações, deixando para traz uma primeira geração de trabalhos com foco em tecnologia da informação e comunicação (TIC), uma segunda geração de estudos sobre criação de conhecimento e cultura organizacional, e alcançando a terceira geração dirigida às pesquisas sobre o desenvolvimento de capacidades organizacionais para criação de valor.
Esta nova geração, iniciada na segunda metade dos anos 2000, busca compreender as interconexões e interdependências entre os mecanismos de aprendizagem e a capacidade organizacional para criação de valor (REZGUI; HOPFE;VORAKULPIPAT, 2010).

De acordo com Popper e Lipshitz (1998,2000), os mecanismos de aprendizagem são essenciais para a utilização do conhecimento criado. São 
os mecanismos de aprendizagem que dialogam com os processos e resultados de gestão do conhecimento, principalmente na coleta, análise, armazenagem, disseminação e aplicação do conhecimento na prática organizacional. Os autores ainda destacam que são estes mecanismos promotores do diálogo entre gestão do conhecimento e aprendizagem organizacional que promovem a renovação sucessiva de ações individuais, grupais e organizacionais, influenciando os resultados alcançados pelos diversos níveis organizacionais. E para que estes mecanismos realmente funcionem como propulsores da melhoria contínua do desempenho da organização, faz-se necessária a sua governança. Ou seja, deve-se implantar a Governança da Aprendizagem Organizacional (GovA).

Aprofundando o tema, Fong e Chen (2012) sugerem que, para governar os mecanismos de aprendizagem, as organizações devem basear-se em quatro perspectivas teóricas: visão baseada no conhecimento; visão da capacidade dinâmica; abordagem da governança do conhecimento (GovC); e abordagem da aprendizagem estratégica.

A visão baseada no conhecimento (GRANT, 1996; SPENDER, 1998) considera o conhecimento como um recurso estratégico, o qual é criado e compartilhado ao mesmo tempo que é evidenciado em suas aplicações nas atividades e processos de negócios para ter um efeito favorável no desempenho da organização.

A visão da capacidade dinâmica (LICHTENTHALER; LICHTENTHALER, 2009; EISENHART; MARTIN, 2000; TEECE， 2007; ZOLLO;WINTER,2002) refere-se a capacidade da empresa de administrar dinamicamente sua base de conhecimento ao longo do tempo, reconfigurando e realinhando os processos de investigação e prospecção de novos conhecimentos (exploration), e o aproveitamento e a retenção (exploitation) deste conhecimento na organização e em sua rede (LICHTENTHALER; LICHTENTHALER, 2009).

Entende-se que, a abordagem da GovC (FOSS, 2007) destaca que os mecanismos de governança dos conhecimentos organizacionais devem ser intencionalmente implantados para influenciar e apoiar os comportamentos e o envolvimento dos membros da organização nos processos de conhecimento, com a intenção de mitigar os custos e os riscos das transações de transferência do conhecimento nas redes inter e intraorganizacionais.

Por fim, a abordagem da aprendizagem estratégica (KAPLAN; NORTON, 1996; PIETERSEN, 2010; STACEY, 2003) ocorre em ambientes onde emergem frequentemente novas ameaças, oportunidades e rápidas mudanças, o que leva as organizações a aprender de maneira a se adaptar, a se desenvolver, ou a transmutar seus modelos mentais para reestruturar seus caminhos.

Com base na literatura de gestão estratégica, Fong e Chen (2012) formularam uma estrutura denominada por eles de Framework de Asserções da Visão de Capacidades Dinâmicas e Aprendizado Estratégico sobre a Gênese e Evolução da Capacidade de Gestão do Conhecimento. Este framework é constituído pelas inter-relações dos mecanismos de governança do conhecimento, dos processos de conhecimento e dos resultados de desempenho organizacional,evidenciando a eficácia dos mecanismos de aprendizagem e da capacidade de gestão do conhecimento para a criação de valor e sustentação de vantagens competitivas ao longo do tempo (Figura 1). 
Figura 1: Framework de asserções da visão de capacidades dinâmicas e aprendizado estratégico sobre a gênese e evolução da capacidade de gestão do conhecimento

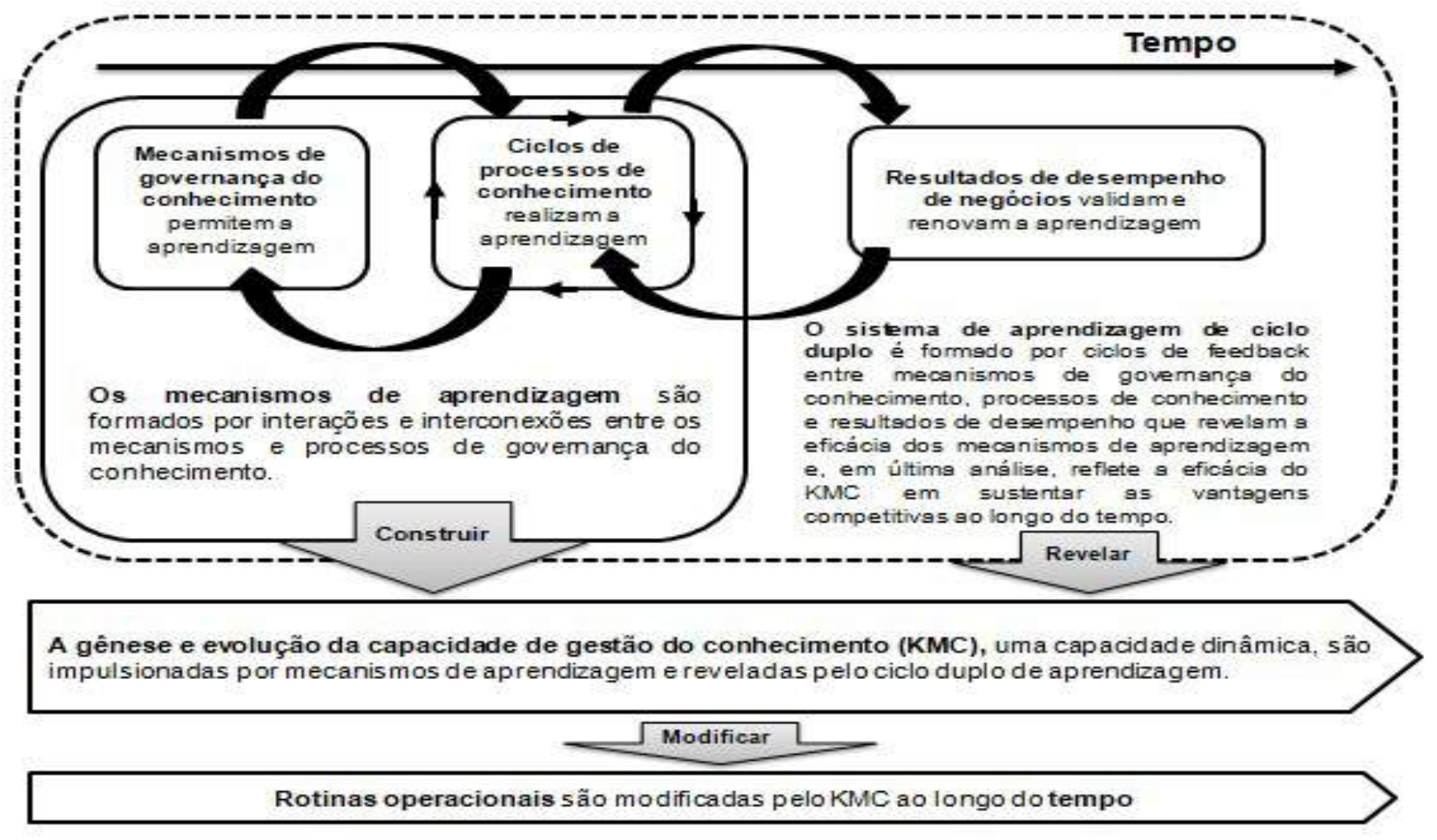

Fonte: Chen e Fong (2013)

$\mathrm{Na}$ leitura deste framework, os mecanismos de governança do conhecimento e processos de conhecimento interagem uns com os outros e formam mecanismos de aprendizagem (ABELL; FELIN; FOSS, 2008; FOSS, 2007; LICHTENTHALER;LICHTENTHALER, 2009; TEECE,2007; NOOTEBOOM,2009), que guiam e conduzem a gênese e a evolução da capacidade de gestão do conhecimento em uma capacidade dinâmica especial de uma empresa (EISENHART; MARTIN, 2000, ZOLLO; WINTER, 2002; LICHTENTHALER; LICHTENTHALER, 2009).

Masquais seriam os mecanismos de Governança do Conhecimento? Na literatura, são encontradas duas abordagens, uma centralizada nas pessoas e outra na tecnologia (ANUMBA, 2009; SCHOLL et al., 2004). Os mecanismos centrados nas pessoas representam os mecanismos que encorajam a inovação, a liderança, as orientações estratégicas e a comunicação.
Para compreender estes mecanismos, outros autores foram identificados. As inovações como "força motriz" e como influência para a criação de novos conhecimentos (CHEN;MOHAMED, 2008). A liderança adequada a transmissão da visão de Gestão do Conhecimento para diferentes níveis organizacionais (CHEN;MOHAMED, 2008). Orientação estratégica para a disseminação do conhecimento (SUBRAMANIAM; VENKATRAMAN, 2001) e para a utilização desse conhecimento na produção de novos conhecimentos (NONAKA; TAKEUCHI, 2004). As comunicações, abertas, flexíveis e eficientes (CHEN; MOHAMED,2008),para criar cultura e clima de estímulo à criação, compartilhamento e (re)uso do conhecimento para resolução de problemas, gerenciamento de mudanças e melhoria da capacidade organizacional (EGBU, 2006; ALAVI; LEIDNER, 2001; GOLD; MALHOTRA; SEGARS, 2001). 
$\mathrm{E}$ os mecanismos tecnológicos representam os mecanismos que ajudam a desenvolver e aplicar sistemas de Tecnologias de Informação e Comunicação, como infraestrutura tecnológica e sua capacidade de responder ao ambiente de trabalho cada vez mais dinâmico para minimizar o custo da solução de problemas, reduzir a probabilidade de repetição de problemas e aumentar a capacidade de inovação (ALAVI; LEIDNER, 2001; CHEN; MOHAMED, 2008; TSERNG; LIN, 2004).

Para autores como Chen e Mohamed (2007); Gold, Malhotra e Segars (2001) e Fong e Chen (2012), a GovC é dependente de quatro processos cíclicos de conhecimentos organizacionais, que são: capacidade de resposta ao conhecimento (CRC), aquisição de conhecimento (AC), disseminação do conhecimento (DC) e utilização do conhecimento (UC).

Especificando os processos, a CRC (CHEN; MOHAMED, 2007) envolve as atividades de conhecimento em resposta aos diversos tipos de informações e conhecimentos a que a organização tem acesso, seja externa ou internamente. A AC, discutida por diferentes
autores(CHEN;MOHAMED,2007;GOLD; MALHOTRA; SEGARS, 2001) abrange a aquisição de conhecimento externo e a criação de novos conhecimentos com base nos conhecimentos já existentes. A DC denota o processo de criação e sustentação de estruturas, sistemas e conteúdos interativos para compartilhamento e retenção de conhecimento na organização (CHEN; MOHAMED, 2007). E, por fim, a UC retrata a utilização do capital intelectual pela organização, especialmente o novo conhecimento tacitamente adquirido pelo colaborador (CHEN; MOHAMED, 2007; GOLD; MALHOTRA; SEGARS, 2001).

Outro mecanismo constitutivo da GovA, segundo Zollo e Winter (2002), são aqueles processos de melhoria relacionados ao ciclo de evolução do conhecimento, o qual faz uso de três mecanismos de aprendizagem: acumulação de experiência, articulação do conhecimento e codificação do conhecimento, por meio de rotinas, compartilhamento e documentação do conhecimento (ZOLLO;WINTER,2002; GAVETTI;LEVINTHAL,2000). Neste ciclo de evolução do conhecimento, as cinco fases ocorrem como mostrado na Figura 2.

Figura 2: Fases do ciclo de evolução do conhecimento

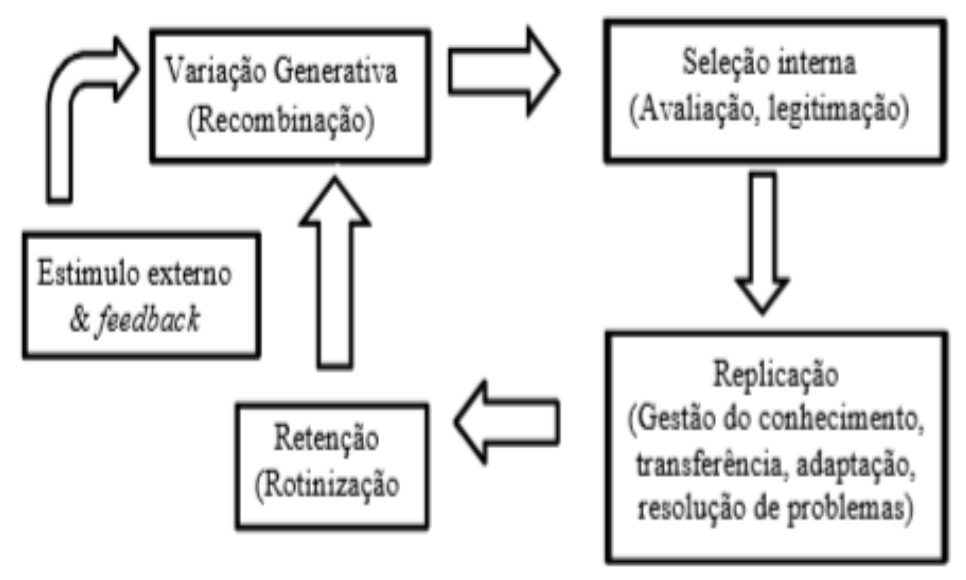

Fonte: Zollo e Winter (2002, p. 343) 
Na figura,identifica-se que um estímulo externo (ou feedback) origina a possibilidade de variação generativa, que acontece quando a ideia principal ou o novo insight torna-se explícito para o debate, gerando a solução de um determinado problema. Essa variação generativa de soluções percorre assim, por um processo de seleção interna, destinadas a avaliar o seu potencial para melhorar a eficácia das rotinas existentes ou a oportunidade de formar novas (NONAKA, 1994), no qual a solução será avaliada e legitimada. Uma vez legitimada, ocorre a fase de replicação do conhecimento através da difusão das mudanças aprovadas dentro da empresa, na qual existe transferência de conhecimento utilizando-se de mecanismos de governança do conhecimento e adaptações para a resolução de problemas, gerando assim novas soluções.
Devidamente replicada, a solução é rotinizada, por meio de novas rotinas ou modificação das já existentes. As rotinas sofrem variações no decorrer do tempo e retroalimentam o processo de conhecimento (ZOLLO;WINTER,2002).

Por fim, estes três mecanismos de aprendizagem - acumulação de experiência, articulação de conhecimento e codificação de conhecimento - constituem o ciclo de evolução do conhecimento, de tal maneira a acumular e renovar o conhecimento, bem como estabelecer novos conhecimentos para as rotinas organizacionais. Para tal, este ciclo busca soluções para as necessidades desconhecidas pela organização e converte estas soluções em rotinas (ZOLLO; WINTER, 2002).

\section{PROCEDIMENTOS METODOLÓGICOS}

A pesquisa relatada neste artigo pode ser considerada de abordagem qualitativa, de cunho teórico, com objetivos exploratórios e descritivos, por meio bibliográfico (MINAYO, 1998; MARCONI;LAKATOS,2009). Foi realizada uma revisão bibliográfica sistemática da literatura, respeitando-se os métodos da revisão integrativa para alcançar maior abrangência sobre o tema. A partir da análise integrativa, realizou-se a síntese sobre GovA, possibilitando a análise do conhecimento científico produzido ao longo do tempo sobre o tema investigado, traçando-se um panorama de modo a conhecer a sua evolução.

No que se refere ao planejamento da revisão, foi elaborado o protocolo da pesquisa bibliográfica, definindo-se a pergunta da revisão, os critérios de inclusão e exclusão e as estratégias de buscas nas bases de dados. A questão de pesquisa
- quais são os mecanismos da Governança da Aprendizagem Organizacional? - foi elaborada com vista a identificar os estudos sobre o termo Governança da Aprendizagem na perspectiva organizacional.

Realizou-se o levantamento nas bases de dados multidisciplinares e internacionais Scopus e Web of Science, devido à abrangência de documentos em diversas áreas (interdisiciplinaridade), aumentando, assim, as chances de obter o maior número de trabalhos referentes à Governança de Aprendizagem.

Quanto ao critério de inclusão, foram consideradas as publicações que se referiam aos descritores no título, nos resumos e nas palavras-chaves. Foram combinados os descritores "governance oflearning" ou "learning governance" ou "governance learning" e "mechanisms". 
Foram excluídas: 3 publicações que não ofereciam acesso ao artigo completo ou gratuito (KOBAYASHI; ONISHI; JEONG, 2014; WIXTED, 2011; BELYAKOV; IVANOVA, 2008); 15 publicações que relacionavam os termos mecanismos, governança e aprendizagem separadamente (MCNUTT; RAYER, 2018; KELLY; ELLIS; FLAMERY, 2018; HEYLIGHEN, 2017; VITTORIA; NAPOLITANO, 2016; CARLSON; PALMER, 2016; GARDNER et al.,2016; RIVERET et al., 2014; ROUILLARD et al., 2013; ESPALLARDO; OREJUELA; PEREZ, 2011; NEOGI; CORDELL, 2010; LOF,2010; SPANGENBERG,2008; CHANG; UDEN,2008;MAYERS et al.,2006a; MAYERS et al.,2006b), 4 publicações que não apresentavam o termo mecanismos, somente o construto "governança da aprendizagem"(GNERRE,1996; CARRHILL,1996;FOTHERINGHAM,2013; SOLOMON,GUSMANO,MASCHKE,2016) e 4 publicações que não apresentavam os termos mecanismos e governança e aprendizagem eram abordados separadamente (SCHEFER, 2019; JONG; KUPPER; BROERSE, 2018; TRAN et al., 2017; BOSSERT; HARTOG, 2015). Na pesquisa não foram excluídas as publicações quanto à data de publicação, porém a seleção de documentos compreendeu até o mês de julho de
2019. Destaca-se que nenhuma publicação foi excluída antes de ter sido lida e analisada.

Ao total, foram identificadas nas bases de dados consultadas 38 publicações que utilizam no título, palavras chave ou resumo, os descritores "governance oflearning" or "learning governance" or "governance learning" and "mechanisms", sendo encontradas 14 publicações na base Scopus e 24 na Web of Science. Em seguida, estes documentos foram exportados para o software EndNote X7, que auxiliou na análise. Entre os documentos retornados, foram identificados e excluídos 11 por estarem duplicados, totalizando 27 documentos elegíveis para análise.

Sobre as 38 publicações, foi realizada uma análise bibliométrica para a compreensão da evolução da produção científica sobre governança da aprendizagem para identificar a quantidade de publicações por ano, autores, países e universidades que abordam o termo. Na sequência, foram lidos os 27 documentos. Destes, foram excluídas 26 publicações. Com 1 documento selecionado nas bases de dados, foram adicionados outros 27 citados pelo artigo selecionado, pois estes autores os julgaram ser relevantes. A Figura 3 apresenta os procedimentos metodológicos adotados.

Figura 3: Procedimentos metodológicos adotados e seus resultados

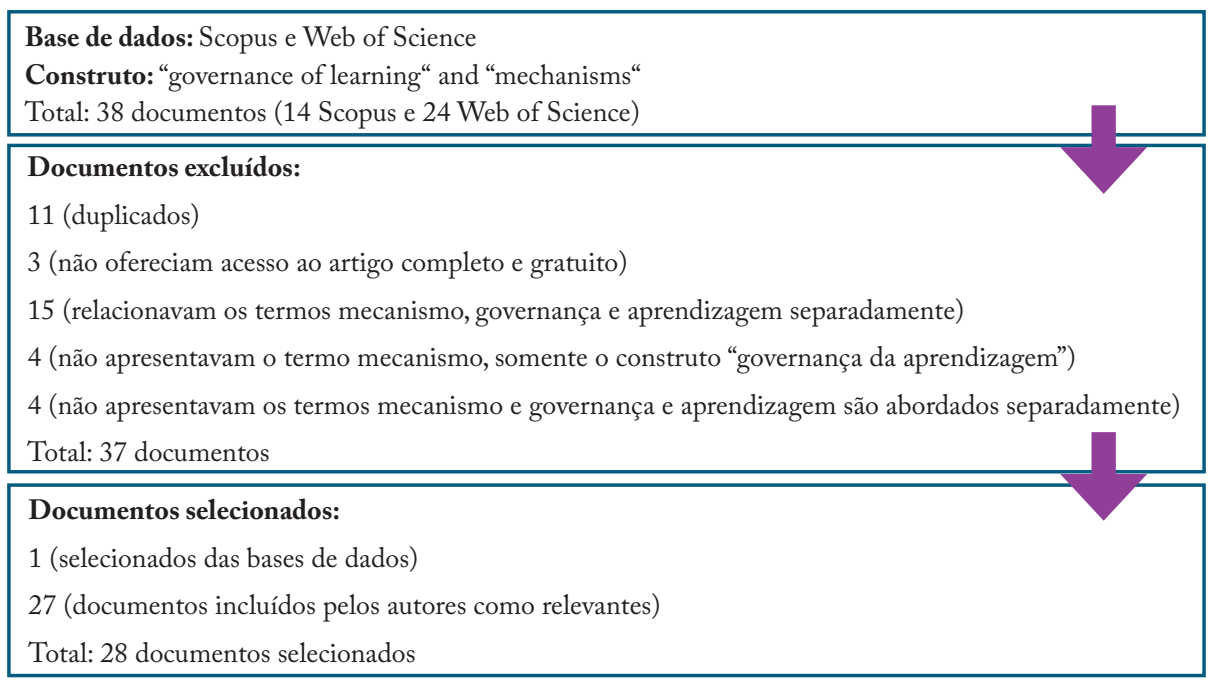

Fonte: Das autoras (2019) 
Entre as publicações complementares, foram acrescentados às 28 publicações os artigos de Belle (2016), Chen e Fong (2013), Pemsel e Muller (2012) e Vitry e Chia (2015) por se relacionarem à GovA, resultando em 32 publicações analisadas em profundidade e, por isso, se configurando na seleção do estudo para a análise integrativa.

\section{ANÁLISE BIBLIOMÉTRICA SOBRE GOVERNANÇA DA APRENDIZAGEM}

Esta seção visa apresentar análise bibliométrica dos 38 documentos encontrados quanto aos descritores utilizados nas buscas nas bases de dados: "governance of learning" or "learning governance" or "governance learning" and "mechanisms" (Quadro 1).

Quadro 1: Distribuição de publicações por base de dados

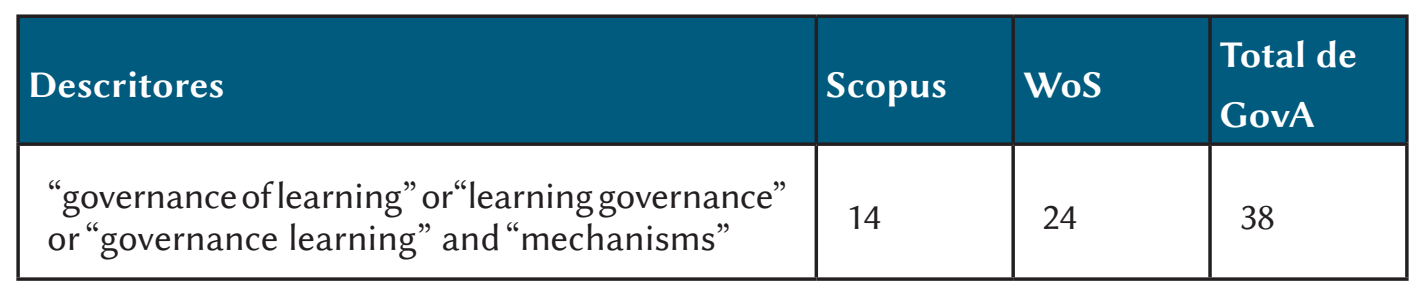

Fonte: Das autoras (2019)

O que se pode perceber em relação a publicação do assunto mecanismos de Governança da Aprendizagem, por ano nas bases de dados, é que apesar de haver poucas variações nas publicações, apresenta uma crescente produtividade sobre o tema em 2016, seguido dos anos de 2010 e 2008 com respectivamente 4, 3 e 3 publicações (Figura 4). No entanto, trata-se de um assunto que necessita ainda de mais pesquisas e aprofundamento, mas que vem crescendo gradativamente.

Gráfico 1: Publicações por ano com os descritores "governance of learning or learning governance or governance learning and mechanisms"

\section{Publicações por ano - Mecanismos de GovA}

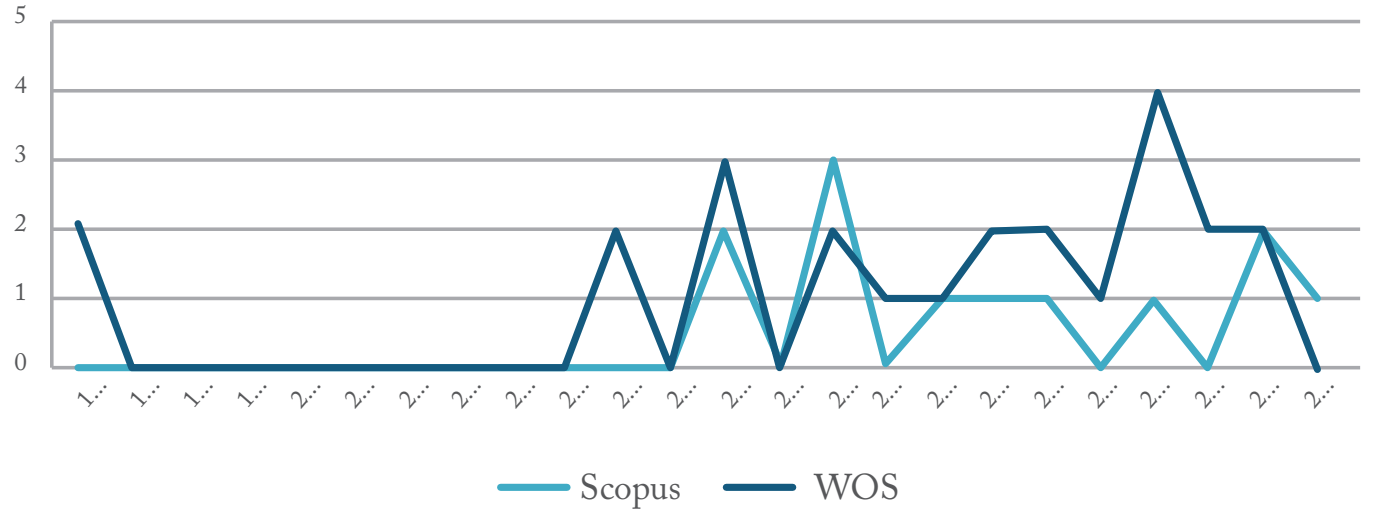

Fonte: Das autoras (2019) 
No gráfico 2, a seguir, são apresentados os autores que, nos bancos de dados Scopus e Web of Science, aparecem como os que mais publicam sobre o assunto utilizando-se de "governance of learning or learning governance or governance learning and mechanisms".

Gráfico 2: Distribuição de autores que mais publicam sobre o termo "governance of learning or learning governance or governance learning and mechanisms"

\section{Publicações por Autores - Mecanismos de GovA}

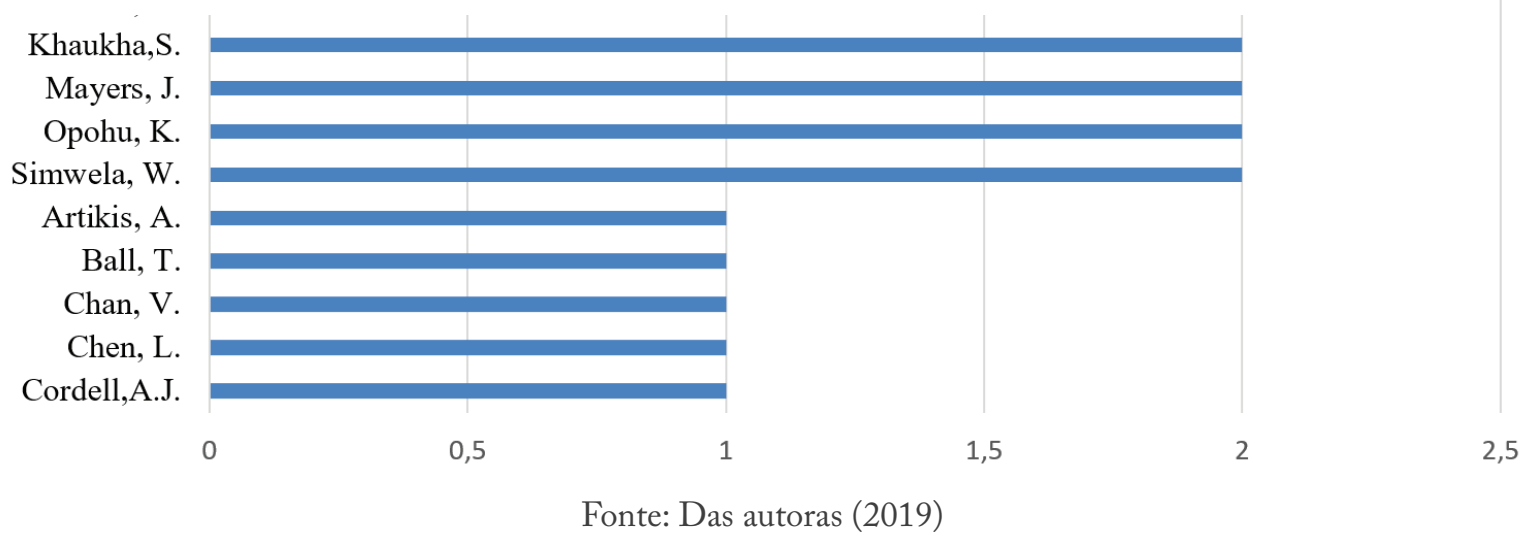

Percebe-se que os autores que mais publicam sobre o assunto são Bila, A., Khaukha, S., Mayers, J., Opohu, K. e Simwela, W., com duas publicações cada um, no ano de 2006. A primeira pesquisadora é professora-assistente na Faculdade de Agronomia e Engenharia Florestal da Universidade Eduardo Mondlane, Maputo, Moçambique. O segundo pesquisador é coordenador de planejamento na Autoridade Florestal Nacional de Kampala, Uganda. O terceiro pesquisador é diretor do Programa de Silvicultura e Uso da Terra do Instituto Internacional para Meio Ambiente e Desenvolvimento de Edimburgo, Reino Unido. O quarto pesquisador é coordenador do Response Civic, em Acra, Gana. E o quinto pesquisador é diretor-adjunto de Florestas no Departamento de Silvicultura de Lilongwe, Malawi.

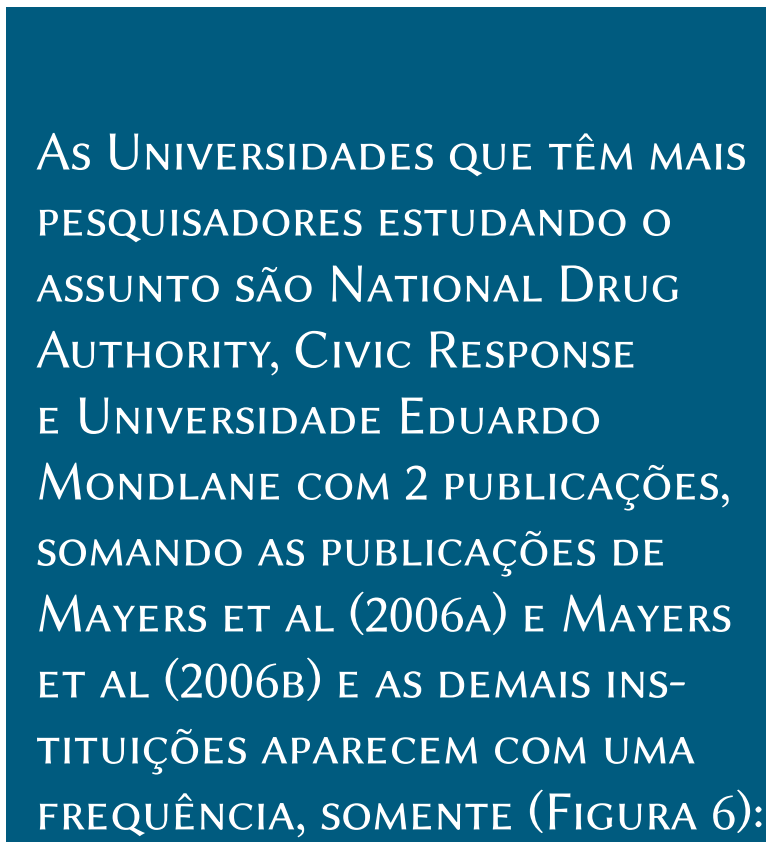


Gráfico 3: Distribuição de publicações por universidades no descritor "governance of learning or learning governance or or governance learning and mechanisms"

\section{Publicações por Universidades - Mecanismos de GovA}

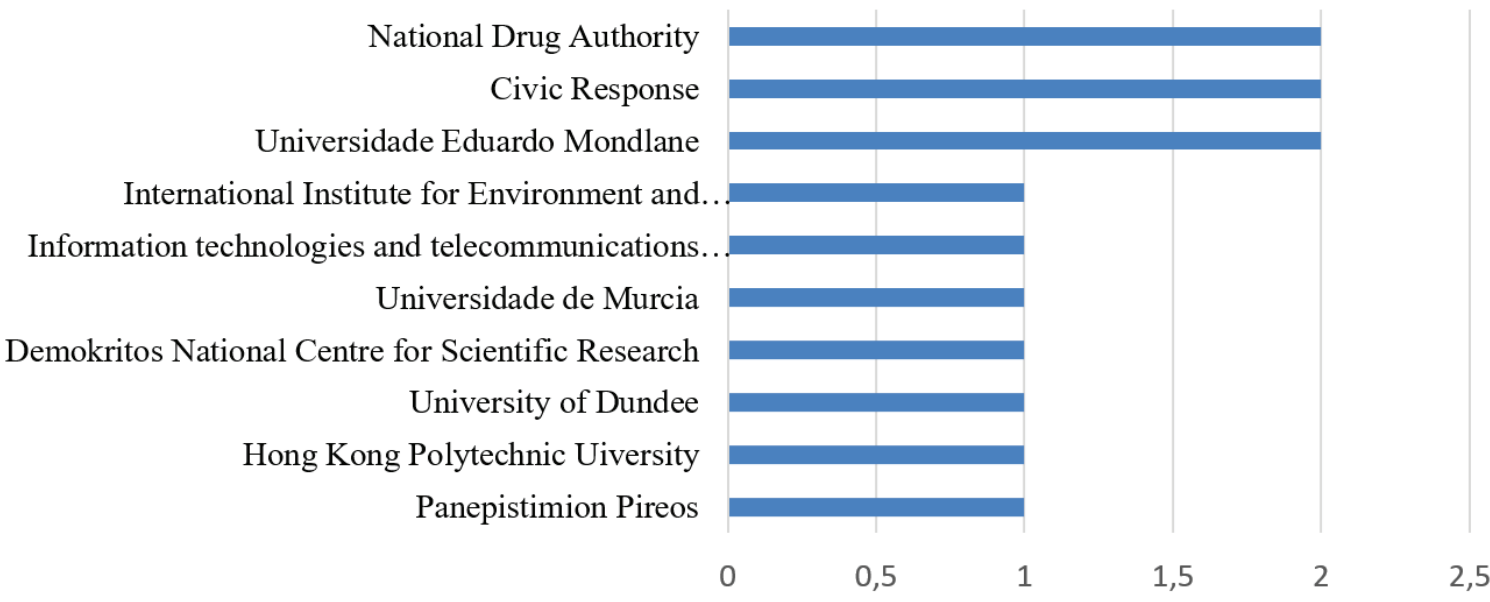

Fonte: Das autoras (2019)

A nação que mais tem demonstrado interesse na publicação sobre o assunto é o Reino Unido, com seis publicações (KELLY; ELLIS; FLANNERY,2018; RIVERET; ARTIKIS; NEPOMUCENO, 2014; ROUILLARD et al., 2013; CHANG; UDEN, 2008; MAYERS et al., 2006a; MAYERS et al., 2006b) (Gráfico 4).

Gráfico 4: Distribuição de publicações por país no descritor "governance of learning or learning governance or governance learning and mechanisms"

\section{Publicações por Países - Mecanismos de GovA}

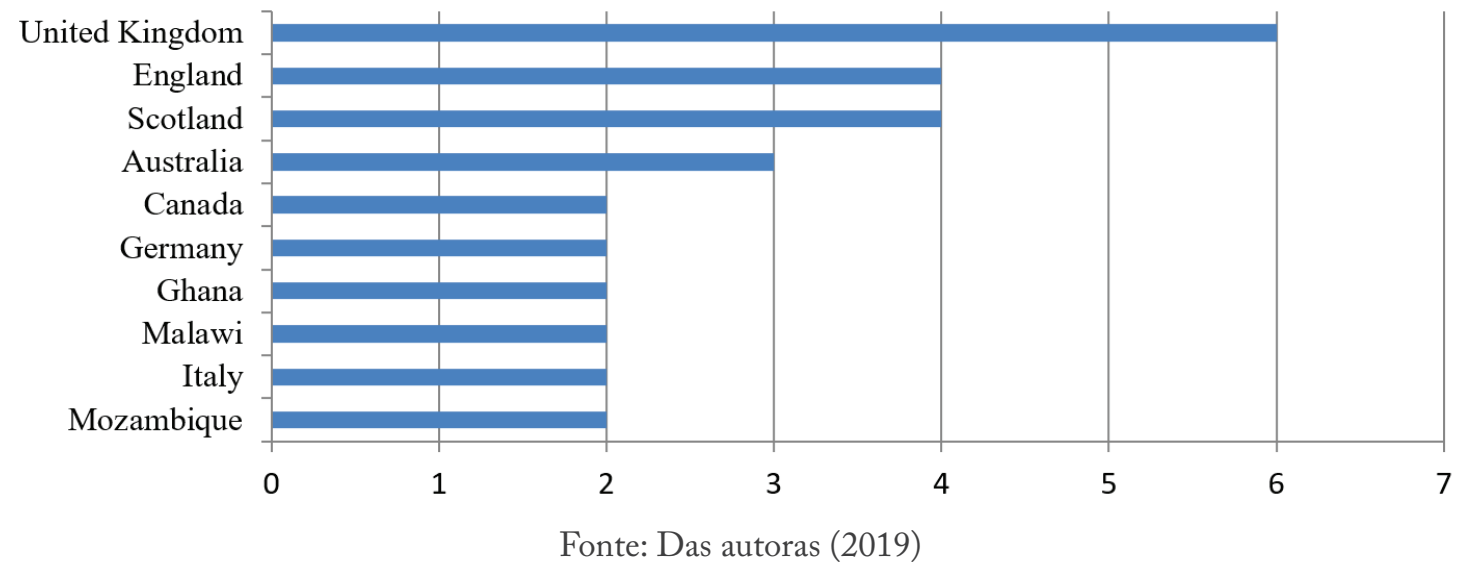

Ao final da análise bibliométrica, pode-se entender que, apesar de identificar 38 publicações sobre o termo e suas variações em inglês, distribuídas entre diversos anos, países, universidades e autores, há a necessidade de novas pesquisas, visto que os estudos sobre os mecanismos de Governança da Aprendizagem Organizacional são ainda recentes e há poucas publicações que aprofundam as discussões. 


\section{ANÁLISE INTEGRATIVA SOBRE GOVERNANÇA DA APRENDIZAGEM}

Para análise integrativa, foram selecionados e analisados 32 documentos, do que se refere aos mecanismos de GovA, foi possível compreender que o assunto abordado neste artigo ainda é muito atual e tem poucas publicações na perspectiva organizacional. Isto é, o assunto tratado é diretamente tratado por apenas um artigo (FONG; CHEN, 2012), indicando uma provável lacuna sobre o assunto.

Nesse único artigo (FONG; CHEN, 2012) e nos demais 31 acrescentados (REZGUI; HOPFE;VORAKULPIPAT,2010; POPPER; LIPSHITZ, 1998, 2000; FONG; CHEN, 2012; GRANT, 1996; SPENDER, 1998; LICHTENTHALER;LICHTENTHALER, 2009; EISENHARDT; MARTIN, 2000; TEECE, 2007; ZOLLO; WINTER, 2002; KAPLAN; NORTON, 1996; PIETERSEN, 2010; STACEY, 2003; CHEN; FONG, 2013; PEMSEL; MULLER, 2012; VITRY; CHIA, 2015; BELLE, 2016; ABELL; FELIN; FOSS, 2008; FOSS, 2007; NOOTEBOOM, 2009; ANUMBA, 2009; SCHOLL et al., 2004; CHEN; MOHAMED, 2007, 2008; SUBRAMANIAM; VENKATRAMAN, 2001; NONAKA;TAKEUCHI,2004; EGBU,
2006; ALAVI; LEIDNER, 2001; GOLD; MALHOTRA; SEGARS, 2001; TSERNG; LIN,2004; GAVETTI;LEVINTHAL,2000; NONAKA,1994), foi possível identificar quais são os mecanismos fundamentais para governar a aprendizagem organizacional.

Foi identificada a proposição de três mecanismos de GovA: a GovC, a GC e o Ciclo de Conhecimento.AGovernançadoConhecimento (GovC) dividido em mecanismos organizacionais e tecnológicos de GovC (FONG; CHEN, 2012; CHEN; MOHAMED, 2008; SUBRAMANIAM; VENKATRAMAN, 2001; NONAKA; TAKEUCHI, 2004; EGBU, 2006, ALAVI; LEIDNER, 2001; GOLD; MALHOTRA; SEGARS, 2001), a Gestão do Conhecimento (FONG; CHEN, 2012; CHEN; MOHAMED, 2007; GOLD; MALHOTRA; SEGARS, 2001; ZOLLO; WINTER, 2002; NONAKA; TAKEUCHI, 2004), e o Ciclo de evolução do conhecimento (ZOLLO;WINTER,2002; NONAKA,1994; NONAKA; TAKEUCHI, 1995).

Em resumo, com base na literatura estudada, os mecanismos constitutivos da Governança da Aprendizagem são os seguintes:

Quadro 2: Mecanismos constitutivos de GovA

\begin{tabular}{|l|l|l|}
\hline \multirow{2}{*}{$\begin{array}{l}\text { Mecanismos de } \\
\text { Governança da }\end{array}$} & $\begin{array}{l}\text { Fong e Chen, 2012; Chen e Mohamed, 2008; } \\
\text { Conhecimento }\end{array}$ & $\begin{array}{l}\text { Subramaniam e Venkatraman, 2001; Nonaka e } \\
\text { Takeuchi, 2004, Egbu, 2006; Alavi e Leidner, 2001; } \\
\text { Gold, Malhotra, Segars, 2001. }\end{array}$ \\
\cline { 2 - 3 } $\begin{array}{l}\text { Aprendizagem } \\
\text { Organizacional }\end{array}$ & $\begin{array}{l}\text { Gestão do } \\
\text { Conhecimento }\end{array}$ & $\begin{array}{l}\text { Malhotra, Segars, 2001; Zollo e Winter, 2002; Nonaka } \\
\text { e Takeuchi, 2004. }\end{array}$ \\
\cline { 2 - 3 } & $\begin{array}{l}\text { Ciclo de Evolução } \\
\text { do Conhecimento }\end{array}$ & $\begin{array}{l}\text { Zollo e Winter, 2002; Nonaka, 1994; Nonaka e } \\
\text { Takeuchi,1995. }\end{array}$ \\
\hline
\end{tabular}

Fonte: Das autoras (2019) 
Por fim, identificou-se que são necessários três mecanismos como Governança do Conhecimento, Gestão do Conhecimento e Ciclo de Evolução do Conhecimento para que a Governança processe a aprendizagem organizacional de maneira a promover o desenvolvimento da organização (Figura 4).

Figura 4: Mecanismos constitutivos de GovA

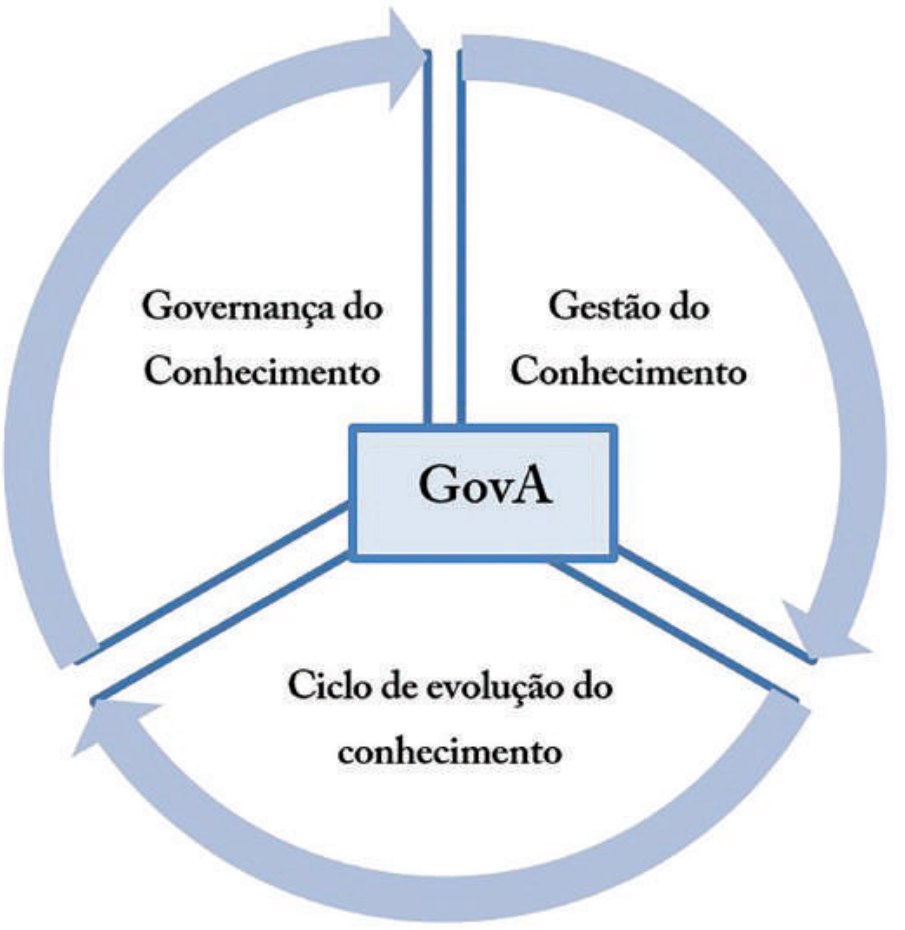

Fonte: Das autoras (2019)

\section{CONCLUSÃO}

Para alcançar o objetivo proposto neste estudo, foram identificados os mecanismos constitutivos da Governança da Aprendizagem sob a perspectiva organizacional propostos pela literatura científica disponível nas bases de dados Scopus e Web of Science, utilizando-se de uma análise bibliométrica e integrativa com abordagem qualitativa e exploratória.

Com base na literatura estudada para análise integrativa, identificou-se por meio dos seus mecanismos, que a GovA é fundamental para melhorar continuamente os processos de aprendizagem organizacional ( $\mathrm{AO})$, de governança do conhecimento (GovC) e de gestão do conhecimento (GC) de maneira a gerar aprendizagem e conhecimentos individuais, grupais e organizacionais para a melhoria do desempenho organizacional. Para tal, é necessária a presença dos mecanismos GovC, GC e ciclo de evolução do conhecimento para governar a aprendizagem organizacional.

Esta pesquisa vem contribuir para a mitigação da lacuna de conhecimento sobre o tema, visto que foi identificada a escassez de estudos direcionados ao construto GovA. Sugere-se a continuidade do estudo, com a realização de novas pesquisas empíricas e teóricas sobre os três mecanismosidentificados e as suas interrelações. 


\section{LEARNING \\ GOVERNANCE MECHANISMS (GovLEARN)}

\begin{abstract}
In order to narrow the gap in studies on GovLearn, this article aims to describe the constitutive mechanisms of Learning Governance from an organizational perspective. The research has a qualitative approach, of a theoretical nature, with exploratory and descriptive objectives, through bibliography, with the support of a systematic review of the literature respecting the methods of the integrative review. As a result, it was possible to describe the analysis of scientific knowledge produced about GovLearn over time, in order to trace its evolution. The proposition of three GovLearn mechanisms was identified: Knowledge Governance (KG), Knowledge Management (KM) and the Knowledge Cycle. Based on the systematic review carried out, it can be said that, in the databases studied, this research contributes to the mitigation of the knowledge gap on the subject.
\end{abstract}

$\quad * * *$
KEY-WORDS: Knowledge
management. Organizational
learning. Knowledge
governance. Governance of
learning.

\section{REFERÊNCIAS}

ABELL, P.; FELIN, T.; FOSS, N. Building microfoundations for the routines, capabilities, and performance links. Management Decision Economics, 29 , n. 6, p. 489-502, 2008.

ALAVI, M.; LEIDNER, D. E. Review: knowledge management and knowledge management systems: conceptual foundation and research issues. MIS Quarterly, v. 25, n. 1, p. 104-136, 2001.

ANUMBA, C. J. Towards next-generation knowledge management systems for construction sector organisations. Construction Innovation: Information, Process, Management, v. 9, n. 3, p. 245-249, 2009.

BELLE, S. Organizational learning? Look again. Learning Organization, v. 23, n. 5, p. 332-341, 2016.

CARLSON, A.; PALMER, C. A qualitative metasynthesis of the benefits of eco-labeling in developing countries. Ecological Economics, v. 127, p. 129-145, 2016.

BELYAKOV, S. A.; IVANOVA, A. A Construction of lifelong learning governance system: key aspects. Journal University Management: Practice and Analysis, p. 5-16, 2008.

BOSSERT, J.; HARTOG, E. M. Den. Managing Human Capital from an Organizational Learning Perspective: Commitment to Creating Public Value. In: INTERNATIONAL CONFERENCE ON PUBLIC ADMINISTRATION, 11., 2015. Proceedings... 2015.

CARRHILL, R. Literacy, Power and democracy in Mozambique: The governance os learning from colonization to the present-Marshall. International Journal of educational development, v. 16, n. 1, p. 103103, 1996.

CHANG, V.; UDEN, L. Governance for e-learning ecosystem. In: INTERNATIONAL CONFERENCE ON DIGITAL ECOSYSTEMS AND TECHNOLOGIES, 2., 2008. Proceedings... IEEE-DEST, 2008.

CHEN, L.; MOHAMED, S. Empirical study of interactions between knowledge management. Engineering, Construction and Architectural Management, v 14, n. 3, p. 242-260, 2007.

CHEN, L.; MOHAMED, S. Impact of the internal business environment on knowledge management within construction organizations. Construction Innovation: Information, Process, Management, v. 8, n. 1, p. 61-81, 2008. 
CHEN, L.; FONG, P. S. W. Visualizing Evolution of Knowledge Management Capability in Construction Firms. Journal of Construction Engineering and Management, v. 139, n. 7, p. 839-851, 2013.

EGBU, C. Knowledge production and capabilities - Their importance and challenges for construction organizations in China. Journal of Technology Management in China, v. 1, n. 3, p. 304-321, 2006.

EISENHART, K. M.; MARTIN, J. A. Dynamic capabilities: What are they? Strategic Management Journal, v. 21, n. 10-11, p. 1105-1121, 2000.

ESPALLARDO, M. H.; OREJUELA, A. R.; PÉREZ, M. S. Inter-organizational governance, learning and performance in supply chains. Supply Chain Management, v. 15, n. 2, p. 101-114, 2010.

FONG, P. S. W; CHEN, L. Governance of Learning Mechanisms: Evidence from Construction Firms. Journal Construction and Engineering Management, $\mathrm{v}$. 138, n. 9, p. 1053-1064, 2012.

FOSS, N. J. The emerging knowledge governance approach: Challenges and characteristics. Organization, v. 14, n. 1, p. 29-52, 2007.

FOTHERINGHAM, D. Confident to seek help: The development of skill and judgement in nurse practitioners. A mixed methods study. Nurse Education Today, v. 33, n. 7, p. 701-708, 2013.

GARDNER, A. et al. Educating for health service reform: Clinical learning, governance and capability - A case study protocol. BMC Nursing, v. 15, n. 1, p. 32, 2016.

GAVETTI, G., LEVINTHAL, D. Looking forward and look backward: Cognitive and experiential search. Administrative Science Quartely, v. 45, p. 113137,2000

GNERRE, M. Literacy, Power and democracy in Mozambique: The governance os learning from colonization to the present-Marshall, J.American Ethnologist, v. 23, n. 4, p. 908-909, 1996.

GOLD, A. H.; MALHOTRA, A.; SEGARS, A. $H$. Knowledge management: An organizational capabilities perspective. Journal Management Information Systems, v. 18, n. 1, p. 185-214, 2001.

GRANT, R. M. Toward a knowledge-based theory of the firm. Strategic Management Journal, Winter Spec. n. 17(SI, p. 109-122, 1996.

HEYLIGHEN, F. The offer network protocol: Mathematical foundations and a roadmap for the development of a global brain. European Physical Journal: Special Topics, v. 226, n. 2, p. 283-312, 2017.
KAPLAN, R. S., NORTON, D. P. Strategic learning and the balanced scorecard. Strategy Leadership, v. 24, n. 5, p. 18-24, 1996.

KEEN, M.; MAHANTY, S. Learning in sustainable natural resource management: Challenges and opportunities in the Pacific. Society and Natural Resources: An International Journal, v.19, n. 6, p. 497513, 2006.

KELLY, C.; ELLIS, G.; FLANNERY, W. Conceptualising change in marine governance: Learning from Transition Management. Marine Policy, v. 95, p. 24-35, 2018.

KOBAYASHI, K.; ONISHI, M.; JEONG, $\mathrm{H}$. Entrepreneur urban policies and regional learning governance. Agglomeration, Clusters and Entrepreneurship: Studies in Regional Economic Development, p. 103-131, 2014.

JONG, I. M. DE; KUPPER, F.; BROERSE, J. Unscripted Responsible Research and Innovation: Adaptive space creation by an emerging RRI practice concerning juvenile justice interventions. Life Sciences, Society and Policy, v. 14, n. 1, 2018.

LICHTENTHALER, U.; LICHTENTHALER, E. A capability-based framework for open innovation: Complementing absorptive capacity. Journal of Management Studies, v. 46, n. 8, p. 1315-1338, 2009.

LÖF, A. Exploring adaptability through learning layers and learning loops. Environmental Education Research, v. 16, n. 5-6, p. 529-554, 2010.

MAYERS, J. et al. Forest governance and social justice: Practical tactics from a learning group approach in Africa. International Forestry Review, v. 8, n. 2, p. 201-210, 2006a.

MAYERS, J. et al. Forest governance and social justice: Practical tactics from a learning group approach in Africa. International Forestry Review, v. 8, n. 1, p. 101-109, 2006b.

MARCONI, M. A.; LAKATOS, E. M. Fundamentos de metodologia cientifica. São Paulo: Atlas, 2009.

MCNUTT, K.; RAYNER, J. Is Learning Without Teaching Possible? The Productive Tension Between Network Governance and Reflexivity. Journal of Environmental Policy and Planning, v. 20, n. 6, p. 797$812,2018$.

MINAYO, M. C DE S. Pesquisa social: teoria, método e criatividade. 9. ed. Petrópolis: Vozes, 1998.

NEOGI, P. K., CORDELL, A. J. The internet and the need for governance: Learning from the past, coping with the future. Journal of Internet Banking and Commerce, v. 15, n. 2, p. 1-30, 2010. 
NONAKA, I.; TAKEUCHI, H. Theory of organisational knowledge creation. In: TAKEUCHI, H.; NONAKA, I. (Eds.). Hitotsubashi on Knowledge Management. Nova York: Wiley, 2004.

NONAKA, I. A dynamic theory of knowledge creation. Organization Science, v. 5, n. 1, p. 14-37, 1994.

NOOTEBOOM, B. A Cognitive theory of the firm: Learning, governance and dynamic capabilities. Cheltenham: Edward Elgar, 2009.

OXTOBY, C.; MOSSOP, L. Checklists and clinical governance: Learning from the NHS. In Practice, v. 38, n. 8, p. 408-410, 2016.

PEMSEL, S.; MÜLLER, R. The governance of knowledge in project-based organizations. International Journal of Project Management, v. 30, n. 8, p. 865-876, 2012.

PIETERSEN, W. Strategic learning: How to be smarter than your competition and turn key insights into competitive advantage. Hoboken: Wiley, 2010.

PRITCHARD, A. Statistical bibliography or bibliometricas? Journal of Documentation, v. 25, n. 4, p. 348-349. 1969.

POPPER, M., LIPSHITZ, R. Organizational learning mechanisms: a structural and cultural approach to organizational learning. The Journal of Applied Behavioral Science, v. 34, n. 2, p. 161-179, 1998.

POPPER, M., LIPSHITZ, R. Organizational learning: mechanisms, culture, and feasibility. Management Learning, v. 31, n. 2, p. 181-196, 2000.

REZGUI, Y.; HOPFE, C. J.; VORAKULPIPAT, C. Generations of knowledge management in the architecture, engineering and construction industry: An evolutionary perspective. Advanced Engineering Informatics, v. 24, n. 2, p. 119-240, 2010.

RIVERET, R. et al. Self-Governance by Transfiguration: From Learning to Prescription Changes. In: INTERNATIONAL CONFERENCE ON SELF-ADAPTIVE AND SELFORGANIZING SYSTEMS. 2014. Proceedings... 2014.

ROUILLARD, J.J. et al. Policy integration for adaptive water governance: Learning from Scotland's experience. Environmental Science and Policy, v. 33, p. 378-387, 2013.

SHEFER, I. Policy transfer in city-to-city cooperation: implications for urban climate governance learning. Journal of Environmental Policy and Planning, v. 21, n. 1, p. 61-75, 2019.
SCHOLL, W. et al. The future of knowledge management: An international delphi study. Journal Knowlegde Management, v. 8, n. 2, p. 19-35, 2004.

SPANGENBERG, J. H. Second order governance: Learning processes to identify indicators. Corporate Social Responsibility and Environmental Management, v. 15, n. 3, p. 125-139, 2008.

SPENDER, J. C. Pluralist epistemology and the knowlegde-based theory of the firm. Organization, v. 5, n. 2, p. 233-256, 1998.

STACEY, R. D. Strategic management and organizational dynamics: the challenge of complexity. Nova York: Prentice Hall; Financial Times, 2003.

SOLOMON, M. Z.; GUSMANO, M. K.; MASCHKE, K. J. The ethical imperative and moral challenges of engaging patients and the public with evidence. Health Affairs, v. 35, n. 4, p. 583-589, 2016.

SUBRAMANIAM, M.; VENKATRAMAN, N. Determinants of transnational new product development capability: testing influence of transferring and deploying tacit overseas knowledge. Strategic Management Journal, v. 22, n. 4, p. 359-378, 2001.

TEECE, D. J. Explicating dynamic capabilities: the nature and micro-foundations of.(sustainable) enterprise performance. Strategic Management Journal, v. 28 , n. 13, p.1319-1350, 2007.

TRAN, L. T. et al. Hybridity in Vietnamese universities: an analysis of the interactions between Vietnamese traditions and foreign influences. Studies in Higher Education, v. 42, n. 10, p. 1899-1916, 2017.

TSERNG, H. P.; LIN, Y. C. Developing an activitybased knowledge management system for contractors. Automation in Construction, v. 13, n. 6, p. 781-802, 2004.

VITRY, C.; CHIA, E. Governance learning: Building a network around managerial innovations. Studies in Public and Non-Profit Governance, v. 4, p. 275-302, 2015.

VITTORIA, M. P.; NAPOLITANO, P. Large cultural networks and smart specialization: What is new in regional policy diagnostic analytics?. Social Network Analysis and Mining, v. 6, n. 1, p. 46, 2016.

WIXTED, B. A cognitive theory of the firm: learning, governance and dynamic capabilities. Science and Public Policy, v. 38, n. 8, p. 661-662, 2011.

ZOLLO, M.; WINTER, S. G. Deliberate learning and the evolution of dynamic capabilities. Organization Science: Journal Management Science, v. 13, n. 3, p. 339-351, 2002. 


\section{SOBRE OS AUTORES}

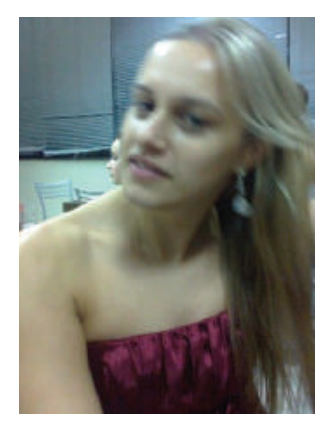

\section{Giselly Rizzatti}

Graduada em Direito pela Universidade do Vale do Itajaí (UNIVALI), com pós-graduação em Direito do Consumidor. Mestre em Administração. Doutora pelo Programa de Pós-Graduação em Engenharia e Gestão do Conhecimento da Universidade Federal de Santa Catarina (EGC/UFSC). Pesquisadora no Grupo de Pesquisa ENGIN (Engenharia da Integração e Governança do Conhecimento) EGC/ UFSC/CNPQ pesquisando o tema Governança da Aprendizagem Organizacional.

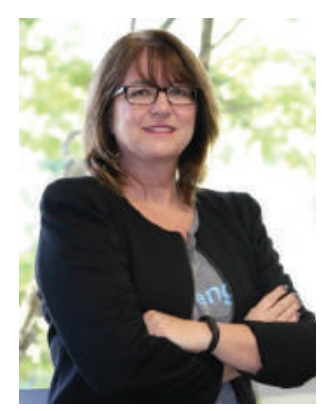

\section{Patricia de Sá Freire}

Professora e Subcoordenadora de Ensino do Departamento de Engenharia do Conhecimento da Universidade Federal de Santa Catarina. Doutora em Engenharia e Gestão do Conhecimento pelo Programa de Pós-graduação em Engenharia e Gestão do Conhecimento/UFSC. Autora de oito livros e mais de 240 artigos científicos publicados em congressos nacionais e internacionais, periódicos e capítulos de livros. Por dois anos seguidos, 2011 e 2012, foi escolhida como um dos cinco executivos de excelência em Gestão do Conhecimento no Brasil pelo MAKE Award Brasil. Atualmente é líder do Grupo de Pesquisa ENGIN (Engenharia da Integração e Governança do Conhecimento) EGC/UFSC/CNPQ e editora-chefe do International Journal of Knowledge Engineering and Management (IJKEM). 